\title{
Fifty years since the discovery of ibuprofen
}

\author{
K. D. Rainsford
}

Published online: 27 November 2011

(C) Springer Basel AG 2011

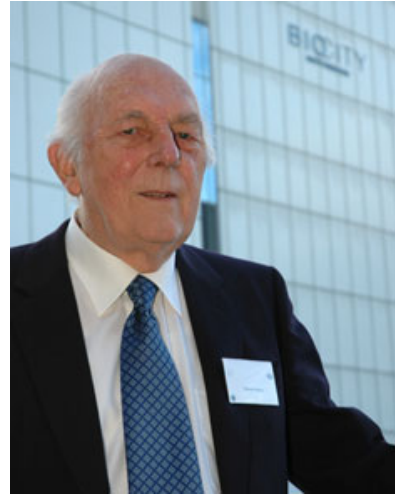

Fig. 1 Professor Stewart Adams outside the BioCity Building in Nottingham which houses part of what were some of the original Boots Company Laboratories, now named in his honour, where development of ibuprofen was undertaken. Photograph kindly provided by Professor Adams and BioCity

It is now 50 years since the discovery of the pharmacological effects of the non-steroidal anti-inflammatory drug (NSAID) ibuprofen, and over 40 years since its introduction to clinical use as a prescription drug for the treatment of arthritic pain and inflammation (Adams 1987; Rainsford 1999, 2003). The initial discovery of the anti-inflammatory effects of ibuprofen in guinea pigs was made on 19 December 1961 by Dr. (now Professor) Stewart Adams, OBE (Fig. 1), the late Dr. John Nicholson (who was the chemist who synthesized the drug), and Mr. Colin Burrows at the Boots Co., Nottingham, UK (Rainsford 1999). The development of ibuprofen by the Boots Company, UK, was

\section{K. D. Rainsford $(\bowtie)$}

Emeritus Professor, Biomedical Research Centre,

Sheffield Hallam University, Howard Street,

Sheffield S11WB, England, UK

e-mail: k.d.rainsford@shu.ac.uk based on the need to have a safer form of aspirin (a "Super Aspirin") without its gastro-intestinal effects, and also without the serious adverse effects of phenylbutazone and corticosteroids; these drugs being the principal antiinflammatory agents available at the time (Adams 1987; Rainsford 1999).

This remarkable discovery was undertaken under what by present day standards that are rather basic laboratory conditions and without much knowledge of the mechanisms of inflammation and biochemical targets to use for drug actions. The discovery of ibuprofen was essentially made on an empirical basis. Indeed, there were many the twists and turns that took place during the discovery and development of ibuprofen from initial humble beginnings. It was only in the early 1970s that the actions of prostaglandins and their actions were established in mediating and regulating inflammation. It took longer still before assays for detecting anti-inflammatory activity based on prostaglandin synthesis inhibition were developed, as well as for the conditions for suitable assays understood, refined and validated for screening potential druggable agents.

It is a great tribute to Dr. (now Professor) Stewart Adams and his colleagues that was through their insight and persistence which enabled the pharmacological activities of ibuprofen to be discovered and the clinical potential to be realized at a time when little was known about techniques for quantifying clinical responses in arthritic and other painful inflammatory conditions.

Initially, after a long period of the synthesis of several hundred compounds Dr. Nicholson prepared, and Dr. Adams and Mr. Colin Burrows screened some 200 salicylate compounds as possible safer and more effective replacements for aspirin. Sadly, this proved unsuccessful although much important pharmacological information was gained from this work (Adams and Cobb 1967). Later, with 
the development and refinement of animal assays other compounds, including over 600 phenoxy-alkanoic acids (originally made as herbicides by Boots), were found to have more potent actions that aspirin or other salicylates in vivo (Nicholson 1982). As shown in the Table 1, there were several compounds that were found to have antiinflammatory activities which were introduced into clinical trials. Among these was the phenyl-acetic acid, RD 10499, and although this was active in rheumatoid arthritis (RA), it unfortunately produced rashes in about $20 \%$ patients, so leading to it being dropped from further clinical investigations. Later the phenyl-acetic acid, ibufenac, which did not produce rashes, was found to be effective in controlling pain and swelling in RA. Unfortunately, it caused severe liver reactions in patients in the UK but, curiously, not in Japan. The reason for this ethnic difference in liver toxicity does not seem to have been determined, but it is interesting, anecdotally, another phenyl-acetic acid NSAID, diclofenac, which also produces liver toxicity in Western patients, has not been observed to produce the same extent as those in Japan.

The occurrence of liver toxicity led to efforts to establish if this was due to accumulation of the drug in the liver. Use of radiolabelled drugs, ibuprofen, it was found that this drug did not accumulate to the extent that was observed with ibufenac. With attention to the pharmacokinetics, gastro-intestinal and liver toxicity, ibuprofen was selected after an extensive programme of drug screening. It may not have been the most potent of the drugs evaluated but it had low toxicity. The recognition since that short half-life NSAIDs with little propensity to accumulate systemically has formed a basis for recognizing the safety of these drugs over those with longer half-lives or accumulation in key organs where untoward reactions may develop (Adams 1988). Indeed, subsequent evaluations have shown that the specific accumulation and persistence of some NSAIDs in their sites of action (i.e. "effect" compartment such as inflamed tissues) relative to their fast clearance from those organs ("side-effect compartments", such as the blood, kidneys and liver) in which toxic reactions may occur with the NSAIDs may explain their relatively low propensity for adverse reactions from drugs like ibuprofen especially at low over-the-counter (OTC) doses (Brune 2007). These investigations have proven an important basis for what is known today about the relative safety of ibuprofen.

The early clinical studies with ibuprofen in rheumatic patients were undertaken with cautious approach to dosage (Rainsford 1999). Safety data became available from some 19,000 patients and this was presented to the UK CSM in support of the case for non-prescription use of ibuprofen. Subsequently, the acceptance of its relative safety led to approval by the UK authorities in 1983 and in 1984 in the USA of low dose $(<1,200 \mathrm{mg} /$ day $)$ for non-prescription
Table 1 Summary of the history of the discovery of ibuprofen at the Boots co., and subsequent worldwide development

1953 Stewart Adams plans search for replacement for aspirin

1955 Development of guinea pig UV erythema as a potential screening assay for new compounds with anti-inflammatory activity

1956

Initiation of chemical development programme by the late Dr John Nicholson. Initially, screening of $>200$ salicylate compounds-proved no better than aspirin (Adams and Cobb 1967)

1960

Phenoxy-acid, RD 8402, in clinical trial. New strategy: search for analgesic and antipyretic with anti-inflammatory activity

1961 Phenyl acetic acid, RD 10355, active in clinical trials in RA, but rash in $5 / 12$ patients led to it being abandoned.

Anti-erythemic activity of ibuprofen discovered. UK Patent application February 1961; final specification September 30, 1964.

1962

Iso-butyl-phenyl acetate, Ibufenac, active clinically in RA, with no rash. In 1968, withdrawn in UK because of liver toxicity, but this was not found in Japanese patients. Liver toxicity related to accumulation of radiolabelled drug in liver.

1964 Ibuprofen, a phenyl propionic acid, made product candidate: Compared with aspirin 16-32× more potent as anti-inflammatory, $30 \times$ more potent as analgesic (Randall Selitto assay) and $20 \times$ more potent as antipyretic. Little accumulation of radiolabelled ibuprofen and low GI toxicity in dogs.

1969 (February) Ibuprofen launched in UK as Brufen ${ }^{\circledR}$

$1970 \quad$ First Symposium on Ibuprofen at Royal College of Physicians, London

$1974 \quad$ Ibuprofen developed as product candidate in 1967 by Upjohn Co. (USA) approved by FDA and launched in USA as Motrin ${ }^{\circledR}$

1983 Ibuprofen approved by the UK CSM for Over the Counter (OTC, or non-prescription) sale. Launched by Crookes Products Ltd (Boots subsidiary) as Nurofen ${ }^{\circledR}$ August 8, 1983

1984

Ibuprofen approved by US FDA for OTC sale on grounds of proven gastric safety. Whitehall Laboratories (Division of American Home Products) by arrangement with Boots, later marketed ibuprofen as Advil ${ }^{\circledR}$

Present day OTC ibuprofen approved for marketing in 82 countries

OTC sale direct to the public. In addition to its wide availability as an OTC analgesic, ibuprofen is now widely used in many countries, often as a first line treatment for the relief of symptoms of pain, inflammation and fever at both prescription as well as non-prescription dosage.

In the recent years, a large number of mega-trials in the recent years have focussed on the clinical uses, safety and 
pharmacological properties of ibuprofen given at prescription level doses $(<2,400 \mathrm{mg} /$ day $)$ compared with newer NSAIDs (including coxibs). In many of these studies, ibuprofen was used as the reference drug in view of its established safety and efficacy. These studies have shown that ibuprofen has comparable safety and efficacy with that of the newer drugs, for long-term usage $(6+$ months).

Spontaneous reports of adverse events and adverse drug reactions (ADRs) in clinical trails from long-term coxib comparator studies as well as in epidemiological studies show that ibuprofen has relatively low risks for gastrointestinal (GI), hepato-renal and other rarer ADRs compared with other NSAIDs and coxibs (Kean et al. 2008; Rainsford et al. 2008; Rainsford 2009). A relatively low risk of cardiovascular (CV) events has been reported in some, not in all studies, but the risks are in general lower than with some coxibs and diclofenac (Psaty and Furberg 2005; Topol 2005; Antman et al. 2007; Purcell 2007; Strand 2007; Trelle et al. 2011). The possibility that ibuprofen may interfere with the anti-platelet effects of aspirin, though possibly of limited significance, has given rise to caution on its use in patients that are at risk for $\mathrm{CV}$ conditions that take aspirin for preventing these conditions (Cryer et al. 2005: Rainsford 2009).

In addition to ibuprofen having unique pharmacokinetics, it also has a broad spectrum of actions on different inflammatory pathways, aside from its affects on pathways of prostaglandin metabolism (Rainsford 2009). This may also be of considerable significance for the actions of the drug in relation to controlling inflammation but also it has relatively low toxicity. The diversity of non-prostaglandin mechanisms, by which, ibuprofen controls inflammation may reduce the dependency on prostaglandin inhibition for controlling inflammation, so achieving a greater overall balance of the regulation of different pathways and mediators of inflammation. The impact of this may be that the relatively low requirement for inhibiting prostaglandin production may reduce the risks of prostaglandin-related side effects (Rainsford 2003).

Since its initial discovery, ibuprofen has been developed in a wide variety of oral and parenteral formulations for use in a variety of indications (Higton 1999; Massey et al. 2010).

In the past decade or so there has been much commercial and clinical interest in developing and use of combinations of ibuprofen with other drugs (e.g. paracetamol, codeine, caffeine) (Rainsford 2012). The objective of many drug combinations has been to raise the "analgesic ceiling" to achieve greater or more sustained acute pain relief. While in many cases the "jury may still be out" on most of these claims, there are already some indications of some potential therapeutic benefits of the drug combinations in certain painful conditions, while still retaining the relative safety benefits of ibuprofen (at least at OTC dosages). Further investigations of these ibuprofen-drug mixtures may be required to establish optimal conditions for their uses in various indications as well as their relative safety.

Paediatric use of has shown that the drug is relatively safe especially in comparison with paracetamol, and effective as a treatment of acute pain and fever. In some conditions ibuprofen is probably more effective than paracetamol as an antipyretic and analgesic (Beaver 2003; Rainsford 2009; Sullivan and Farrar 2011). A recent clinical assessment of antipyretic use in children showed there are no substantial differences in the safety and efficacy of ibuprofen and paracetamol in the care of a generally healthy child with fever (Sullivan and Farrar 2011). Although hepatotoxicity is reported rarely with paracetamol at recommended doses, there is a concern when there has been overdose. Ibuprofen appears to have longer clinical effect in reducing elevated temperatures than paracetamol (Sullivan and Farrar 2011). Nephrotoxicity with ibuprofen has been reported to be a concern in children with febrile illnesses (Sullivan and Farrar 2011) and the general advice is to watch for dehydration and ensure that patients receive adequate fluids.

At OTC doses ( $<1,200 \mathrm{mg} /$ day $)$ ibuprofen has a comparable safety profile with that of paracetamol. Its antiinflammatory activity is linked to its analgesic effects and this is related to reduction in the ex vivo production in blood of cyclo-oxygenase (COX)-1- and COX-2-derived prostanoids (Blain et al. 2002; Rainsford 2009). Ibuprofen at OTC doses has low possibilities of serious GI events, and little prospect of developing renal and associated $\mathrm{CV}$ events. Ibuprofen OTC does not represent a risk for developing liver injury especially the irreversible liver damage observed with paracetamol and the occasional liver reactions from aspirin (Rainsford 2009). One of the successes with ibuprofen since its introduction in a low dose $(\leq 1,200 \mathrm{mg} /$ day $)$ for OTC use that it has proven relatively safe and effective such that it is now licensed and available in over 80 countries worldwide.

Large-scale clinical and epidemiological studies have shown that ibuprofen OTC is safe, especially in the GI tract than aspirin, and has similar GI safety compared with paracetamol (acetaminophen), yet without the risks of liver toxicity seen with the latter drug (Rainsford 2009). This is not to say that OTC ibuprofen is without adverse effects. However, the occurrence of these untoward actions is rare and well understood and most reactions are reversible upon cessation of the drug (indicating reversibility of toxic mechanisms) (Volans et al. 2003).

Research on the diverse pharmacological properties of ibuprofen, like that of some other NSAIDs, has advanced in parallel with understanding of the cell and molecular 
biology of inflammatory processes, especially those underlying neuro-pathological reactions in pain and neurodegenerative diseases (Townsend and Praticò 2005; Kotilinek et al. 2008) and cancer-related inflammatory reactions (Wynne and Djakiew 2010; Greenspan et al. 2011; Woodman et al. 2011). Consequently, more interest has been shown in the past two decades or so in the potential for ibuprofen to prevent conditions such as Alzheimer's and atherosclerotic dementias, Parkinson's disease and nerve injuries (Townsend and Praticò 2005; Hirohata et al. 2008; Kotilinek et al. 2008), as well as colorectal, mammary, prostate and some other cancers (Harris 2002; Rostrom et al. 2007; Brasky et al. 2010, 2011; Mahmud et al. 2011). While these developments are undoubtedly exciting, there will, however, extensive investigations which will have to be performed to understand when ibuprofen should be employed in the various stages of these chronic and complex conditions and at what dose(s). Indeed, special formulations of ibuprofen may need to be developed to ensure optimal biodisposition of the drug (e.g. localized delivery in the colon in colo-rectal cancer) or prolonged pharmacokinetics for specific applications in different chronic diseases (e.g. in cystic fibrosis) or special patient groups (young and the elderly) whose long-term safe use is required (Rainsford 2012). The extensive interest on the effects of ibuprofen in these nonpain conditions attests to the diversity of the pharmacological effects of ibuprofen as well as differences in comparison with other NSAIDs.

Over the years since the introduction of ibuprofen, it has withstood competition from newer NSAIDs and paracetamol and commands a place in the mainstay of therapy for pain and inflammation. Moreover, there have been a considerable number of NSAIDs that have been withdrawn since the development of this class of drugs from the 1970s (Rainsford 1987) and more recently with some of the coxibs (Topol 2005). It is, therefore, notable that ibuprofen has survived where other drugs have failed and been a leading drug for the relief of symptoms of mild-to-moderate pain in various arthritic and other chronic inflammatory conditions where it has been used as a prescription drug and the relief of various inflammatory pain conditions at OTC doses.

\section{References}

Adams SS (1987) The discovery of Brufen. Chem Br 23:1193-1195 Adams SS (1988) Non-steroidal anti-inflammatory drugs, plasma half-lives, and adverse reactions. Lancet 2:1204-1205

Adams SS, Cobb R (1967) Non-steroidal antiinflammatory drugs. In: Ellis GP, West GB (eds) Progress in medicinal chemistry 5. Butterworths, London, pp 59-133
Antman EM, Bennett JS, Daugherty A, Furberg C, Roberts H, Taubert KA, American Heart Association (2007) Use of nonsteroidal antiinflammatory drugs: an update for clinicians: a scientific statement from the American Heart Association. Circulation 115:1634-1642

Beaver WT (2003) Review of the analgesic efficacy of ibuprofen. Int J Clin Pract Suppl 135:13-17

Blain H, Boileau C, Lapicque F, Nédélec E, Loeuille D, Guillaume C, Gaucher A, Jeandel C, Netter P, Jouzeau JY (2002) Limitation of the in vitro whole blood assay for predicting the COX selectivity of NSAIDs in clinical use. Br J Clin Pharmacol 53:255-265

Brasky TM, Velicer CM, Kristal AR, Peters U, Potter JD, White E (2010) Nonsteroidal anti-inflammatory drugs and prostate cancer risk in the VITamins And Lifestyle (VITAL) cohort. Cancer Epidemiol Biomarkers Prev 19:3185-3188

Brasky TM, Bonner MR, Moysich KB, Ambrosone CB, Nie J, Tao MH, Edge SB, Kallakury BV, Marian C, Goerlitz DS, Trevisan M, Shields PG, Freudenheim JL (2011) Non-steroidal antiinflammatory drugs (NSAIDs) and breast cancer risk: differences by molecular subtype. Cancer Causes Control 22:965-975

Brune K (2007) Persistence of NSAIDs at effect sites and rapid disappearance from side-effect compartments contributes to tolerability. Curr Med Res Opin 23:2985-2995

Cryer B, Berlin RG, Cooper SA, Hsu C, Wason S (2005) Doubleblind, randomized, parallel, placebo-controlled study of ibuprofen effects on thromboxane $\mathrm{B}_{2}$ concentrations in aspirin-treated healthy adult volunteers. Clin Ther 27:185-191

Greenspan EJ, Madigan JP, Boardman LA, Rosenberg DW (2011) Ibuprofen inhibits activation of nuclear $\{$ beta\}-catenin in human colon adenomas and induces the phosphorylation of GSK$3\{$ beta\}. Cancer Prev Res 4:161-171

Harris RE (ed) (2002) COX-2 blockade in cancer prevention and therapy. Humana Press, NJ, Totawa

Higton F (1999) The pharmaceutics of ibuprofen. In: Rainsford KD (ed) Ibuprofen. A critical bibliographic review. Taylor \& Francis, London, pp 55-86

Hirohata M, Ono K, Morinaga A, Yamada M (2008) Non-steroidal anti-inflammatory drugs have potent anti-fibrillogenic and fibrildestabilizing effects for alpha-synuclein fibrils in vitro. Neuropharmacology 54:620-627

Kean WF, Rainsford KD, Kean IRL (2008) Management of chronic musculoskeletal pain in the elderly: opinions on oral medication use. Inflammopharmacology 16:53-75

Kotilinek LA, Westerman MA, Wang Q, Panizzon K, Lim GP, Simonyi A, Lesne S, Falinska A, Younkin LH, Younkin SG, Rowan M, Cleary J, Wallis RA, Sun GY, Cole G, Frautschy S, Anwyl R, Ashe KH (2008) Cyclooxygenase-2 inhibition improves amyloid-beta-mediated suppression of memory and synaptic plasticity. Brain 131:651-664

Mahmud SM, Franco EL, Turner D, Platt RW, Beck P, Skarsgard D, Tonita J, Sharpe C, Aprikian AG (2011). Use of non-steroidal anti-inflammatory drugs and prostate cancer risk: a populationbased nested case-control study. PLoS One 6(1):e16412

Massey T, Derry S, Moore RA, McQuay HJ (2010) Topical NSAIDs for acute pain in adults. Cochr Database Syst Rev (6):CD007402

Nicholson JS (1982) Ibuprofen. In: Bindra JS, Lednicer D (eds) Chronicles of drug discovery, Chapter 7, vol 1. Wiley, New York, pp 149-171

Psaty BM, Furberg CD (2005) COX-2 inhibitors-lessons in drug safety. N Engl J Med 352:1133-1135

Purcell H (2007) Ibuprofen and cardiovascular safety: where do we stand? J R Soc Med 100(Suppl 48):7-10

Rainsford KD (1987) Introduction and historical aspects of the side effects of anti-inflammatory analgesic drugs. In: Rainsford KD, Velo GP (eds) Side effects of anti-inflammatory analgesic drugs, vol 1. MTP Press, Lancaster, pp 3-26 
Rainsford KD (1999) History and development of ibuprofen. In: Rainsford KD (ed) Ibuprofen. A critical bibliographic review, Chapter 1. Taylor \& Francis, London, pp 3-24

Rainsford KD (2003) Discovery, mechanisms of action and safety of ibuprofen. Intl J Clin Pract Suppl (135):3-8

Rainsford KD (2009) Ibuprofen: pharmacology, efficacy and safety. Inflammopharmacology 17:275-342

Rainsford KD (2012) Ibuprofen. Pharmacology, therapeutics and side effects. Springer press, Basel and Heidelberg (in press)

Rainsford KD, Kean WF, Ehrlich GE (2008) Triad (GI, CV, Hepatic) composite toxicity ratings for use in assessing the overall safety of NSAIDs. Intern Med J, 38(Suppl. 2):A32 (Abstract No. ARP71)

Rostrom A, Dube C, Lewin G (2007) Use of aspirin and NSAIDs to prevent colorectal cancer (Internet). Agency for Healthcare Research and Quality (US), Rockville. (Available from http://www.ncbi.nlm.nih.gov/bookshelf/br.fcgi?book=es45)

Strand V (2007) Are COX-2 inhibitors preferable to non-selective nonsteroidal anti-inflammatory drugs in patients with risk of cardiovascular events taking low-dose aspirin? Lancet 370:2138-2151

Sullivan JE, Farrar HC (2011) Section on clinical pharmacology and therapeutics; committee on drugs, fever and antipyretic use in children. Pediatrics 127:580-587
Topol EJ (2005) Arthritis medicines and cardiovascular events"house of coxibs". J Am Med Assn 293(3):366-368

Townsend KP, Praticò D (2005) Novel therapeutic opportunities for Alzheimer's disease: focus on nonsteroidal anti-inflammatory drugs. FASEB J 19:1592-1601

Trelle S, Reichenbach S, Wandel S, Hildebrand P, Tschannen B, Villiger PM, Egger M, Jüni P (2011) Cardiovascular safety of non-steroidal anti-inflammatory drugs: network meta-analysis. Br Med J 342:7086

Volans G, Monaghan J, Colbridge M (2003) Ibuprofen overdose. Int J Clin Pract Suppl (135):54-60

Woodman TJ, Wood PJ, Thompson AS, Hutchings TJ, Steel GR, Jiao P, Threadgill MD, Lloyd MD (2011) Chiral inversion of 2-arylpropionyl-CoA esters by human $\alpha$-methylacyl-CoA racemase 1A (P504S) - a potential mechanism for the anti-cancer effects of ibuprofen. Chem Commun 47:7332-7334

Wynne S, Djakiew D (2010) NSAID inhibition of prostate cancer cell migration is mediated by Nag-1 Induction via the p38 MAPKp75(NTR) pathway. Mol Cancer Res 8:1656-1664 